\title{
Anterior segment OCT evaluation of a caucasian population to assess posterior corneal arc length as a utility for planning endothelial keratoplasty
}

\begin{abstract}
Purpose: To provide the first data of posterior corneal arc length (PCAL) in a British Caucasian population. To assess the correlation of PCAL with white to white distance (WTW), anterior chamber (AC) diameter and depth. To establish whether PCAL measurements may be of use in determining graft diameter in corneal endothelial transplant surgery.

Methods: Patients were recruited from a database of anterior segment optical coherence tomography images (Tomey Casia). Patients were included if their demographic information confirmed that they were White British. Patients were excluded if they had previously had corneal surgery. 192 eyes from 100 patients were used for the study. Together with Tomey, we developed image analysis software to measure PCAL. This was used to measure PCAL, WTW and AC depth and diameter. Statistical analyses were performed using SPSS 14.0.

Results: 54 patients were female (54\%) and 46 were male (46\%). The average age of the patients was 62 (range 22-88). Mean PCAL was $13.38 \mathrm{~mm}$ (95\% CI 13.48 and 13.68 ) (SD $0.71 \mathrm{~mm})$. Mean WTW was $11.74 \mathrm{~mm}(95 \%$ CI 11.6711 .82$)$ (SD 0.51mm). PCAL correlated extremely well with WTW distance (Pearson's $\mathrm{r}=0.924, \mathrm{p}<0.01$ ). Mean WTW to PCAL ratio was 1.16 (1.08-1.21). PCAL also correlated well with ACD (Pearson's r $=0.541$, $\mathrm{p}<0.01$ ) but the ratio of PCAL to ACD was much less consistent than that of PCAL to WTW.

Conclusion: This is the first data documenting PCAL measurements in a White Caucasian population. PCAL correlates closely with WTW and may be extrapolated using a conversion factor of 1.16. PCAL may have a role in sizing of endothelial grafts but further analysis and larger patient numbers are required.
\end{abstract}

Volume 2 Issue 6 - 2015

\author{
Alastair J Stuart,' Alex Shortt, ${ }^{2}$ Romesh I \\ Angunawela ${ }^{3}$ \\ 'Ophthalmic Registrar, Sidcup Hospital, England \\ ${ }^{2}$ Consultant Ophthalmologist, Moorfields Eye Hospital, London \\ ${ }^{3}$ Consultant Ophthalmic Surgeon, Moorfields Eye Hospital, \\ London
}

Correspondence: Alastair J Stuart, Ophthalmic Registrar, Sidcup Hospital, Frognal Avenue, Sidcup, Kent DA 14 6LT, England, Tel 07843005295, Email alastairstuart@nhs.net

Received: August 06, 2015 | Published: August 27, 2015
Abbreviations: PCAL, posterior corneal arc length; WTW, white to white distance; AC, anterior chamber; DSAEK, descemet stripping automated endothelial keratoplasty; OCT, optical coherence tomography

\section{Introduction}

Detailed and quantitative knowledge of anterior segment anatomy is essential when performing modern lamellar corneal surgery which aims to preserve healthy tissue whilst replacing diseased or dysfunctional tissue. Recent evidence suggests that large endothelial grafts used during descemet stripping automated endothelial keratoplasty (DSAEK) result in better graft survival, presumably due to increased numbers of transplanted endothelial cells. ${ }^{1}$ Even if larger graft sizes are desirable for DSAEK, appropriateiate sizing based on individual patient anatomy is necessary to avoid complications such as peripheral anterior synechiae formation, which may cause endothelial cell attrition and subsequent graft failure. Basic slit lamp assessment and per-operative calliper measurement of the cornea or anterior segment are inherently limited to cord length in a spherical structure such as the eye. Optical coherence tomography (OCT) was first reported in $1991^{2}$ and has become an increasingly useful tool in the assessment of patients with ophthalmic conditions. OCT provides an objective, non-contact method of assessing anterior segment anatomy. ${ }^{3}$ It is already used to provide accurate angle analysis in glaucoma patients ${ }^{4}$ and is being developed to provide an intraoperative guide for corneal transplant surgery. ${ }^{5}$

The Zhongshan Angle Assessment Program, a third party software developed in 2008, provided a method of accurately measuring anterior segment parameters from raw OCT image data obtained from a time-domain OCT (Visante, Carl Zeiss Meditec, Jena, Germany). ${ }^{6}$ As part of this work, a novel corneal parameter, posterior corneal arc length (PCAL) was developed. ${ }^{7}$ This is defined as the arc distance of the posterior corneal border between sclera spurs. The anatomical variation of this parameter has been documented in Asian eyes, ${ }^{7-9}$ but to our knowledge has not been described in a Caucasian population.

Previous studies of anterior chamber dimensions, particularly PCAL, used early time domain anterior segment OCT imaging technology. ${ }^{7-9}$ OCT technology has evolved in pursuit of increased resolution and reduced motion artefact. Swept- source OCT (SSOCT) time-encodes wave number by rapidly tuning a narrowband source through a broad optical bandwidth. Swept source OCT has been shown to be more sensitive (when defined by Signal to noise ratio) than time domain OCT. ${ }^{10}$ The aim of this study was to document anterior chamber metrics, and in particular PCAL data in a caucasian 
population using a SS-OCT method. PCAL is of significance in terms of the current trend in treatment of diseases of endothelial dysfunction by endothelial keratoplasty where optimal graft size may improve outcomes.

\section{Methods}

\section{Study population}

Approval for this study was granted by the Research and Development Department of NIHR Biomedical Research Centre at Moorfields Eye Hospital and UCL Institute of Ophthalmology. Patients were scanned prospectively and a database of anterior segment OCT images created. Only caucasian subjects were included in this study. Exclusion criteria included non-caucasian race, any anterior segment pathology and any previous anterior segment surgery. Subjects were recruited from anterior segment and general primary care clinics. 100 subjects were included.

\section{Imaging}

All images were collected using the Tomey Casia (Tomey, Nagoya, Japan) swept source spectral domain anterior segment OCT. Images were collected using the "anterior segment" settings. The Casia OCT produces a three dimensional image based on the principal of "swept source" OCT. The wavelength of the light source is $1310 \mathrm{~nm}$. It produces high resolution imaging of 10um (axial) and 30 um (transverse) resolution and a scan rate of of 30,000 A-scans per second. Rapid scan frequency and the ability to scan the entire anterior segment within 0.3-2.4 seconds, minimizes motion artefact.

\section{Image processing}

We developed novel image analysis software in conjunction with Tomey (Tomey Inc, Nagoya, Japan). This allowed individual raw OCT image data to be exported from the Casia OCT and analyzed. The software utilizes an image processing filter to plot the anterior and posterior corneal curvature allowing PCAL to be measured. Hundreds of points are plotted along the posterior curvature and distance between adjacent points is integrated to give the length of the arc. For all images, the white to white measurement, anterior chamber depth and posterior corneal arc length (distance of the posterior corneal border between the sclera spurs) was recorded. All images were processed and analyzed by the same clinician (AJS).

Table I Summary of results

\section{Statistical analysis}

The distribution of data for anterior chamber depth, posterior corneal arc length and white to white measurements was assessed for normality using the Shapiro-Wilk test. For parametric data means, $95 \%$ confidence intervals and standard deviations are reported. For non-parametric data the median and interquartile range are reported. To validate the data differences between right and left eyes, Wilcoxon test was used for non-parametric paired data, and a paired $t$ test used for parametric paired data. The correlation between posterior corneal arc length and white to white distance was assessed using Pearson's correlation co-efficient. The correlation between posterior corneal arc length and anterior chamber depth was analyzed using Spearman's rank test for all statistical tests an alpha level of 0.05 was considered as significant. All statistical analyses were performed using SPSS 14.0 for Windows.

\section{Results}

Of the 100 patients included, 46 were male $(46 \%)$ and 54 were female $(54 \%)$. The average age was 62 with a ranging from 22 to 88. 92 patients had both left and right eyes included in the study. Of the other 8 patients, 5 were right eyes and 3 were left. These patients only had one eye scanned because the other eye fell into the exclusion criteria (previous anterior segment pathology). Posterior corneal arc length and white to white distance were normally distributed (Shapiro-Wilk test, $\mathrm{p}>0.05$ ). There was no difference between right and left eye measurements for either value (paired t-test). There was no difference in any of the variables between right and left eyes (paired t-test: ACD RE vs LE $p=0.273$; PCAL RE vs LE $p=0.143$, WTW RE vs LE $\mathrm{p}=0.144$ ).

Table 1 Mean PCAL was $13.58 \mathrm{~mm}$ (95\% CI 13.48 and 13.68) (SD $0.71 \mathrm{~mm})$. Mean WTW was $11.74 \mathrm{~mm}(95 \%$ CI 11.67 and 11.82$)$ (SD $0.51 \mathrm{~mm})$. Mean WTW was $11.74 \mathrm{~mm}$ (95\% CI 11.67 and 11.82$)$ (SD $0.51 \mathrm{~mm}$.) Posterior corneal arc length correlated extremely highly with white to white distance (Pearson's $r=0.924, p<0.01$ ). PCAL was always greater than WTW and the mean difference between these measures was $1.82 \mathrm{~mm}$ (range $0.81 \mathrm{~mm}$ to $2.58 \mathrm{~mm}$ ). The ratio of PCAL to WTW distance was extremely consistent with a mean of 1.16 (range 1.08 to 1.21). PCAL also correlated with ACD (Pearson's r $=0.541$, $\mathrm{p}<0.01$ ) but the ratio of PCL to ACD was much less consistent (mean 4.84 , range 3.0 to 7.2 ).

\begin{tabular}{llll}
\hline Variable & Mean $(\mathbf{m m})$ & $\mathbf{9 5 \%}$ Confidence interval (Lower, Upper)(mm) & Standard deviation $(\mathbf{m m})$ \\
\hline Anterior chamber depth & 2.87 & $2.79,2.95$ & 0.57 \\
Posterior corneal arc length & 13.58 & $13.48,13.68$ & $0.7 \mid$ \\
White to white distance & 11.74 & $11.67,11.82$ & $0.5 \mid$
\end{tabular}

\section{Discussion}

This study presents the first data series, to our knowledge, describing anterior segment metrics and posterior corneal arc length measurements in a British caucasian population. These measurements are normally distributed. The average PCAL in our study was $13.58 \mathrm{~mm}$ (SD 0.71). Compared to results of other papers, this value was higher than that in a Chinese ${ }^{8}$ population $(12.92 \mathrm{~mm}$ SD 0.54$)$ and lower than that in Malay $^{7}(13.95 \mathrm{~mm}$ SD 0.51) and South Asian Indian $^{9}(13.85 \mathrm{~mm}$ SD 0.54$)$ populations. We have shown there to be a significant correlation between PCAL and WTW measurements in this British caucasian population. We have also shown there to be a correlation between PCAL and ACD, albeit less consistent. Previous studies into PCAL have been limited to Eastern patient demographics and have not directly compared the correlation between PCAL and WTW. These studies did find a similar correlation between PCAL and $\mathrm{ACD}^{7-9}$ to that found with our data.

The anterior segment OCT used in this study is a swept source spectral domain OCT, and hence the measurements described in this 
study are likely to be more accurate than prior studies that have utilized time domain technology which is less sensitive (due to lower SNR) ${ }^{10}$ The image analysis software that we developed in conjunction with Tomey (Tomey, Nagoya, Japan) allowed accurate measurement of PCAL. As with other optical coherence topographers, the Casia OCT allows accurate measurement of linear anterior segment parameters such as depth and diameter. However, although the Casia registers anterior and posterior corneal and iris contour to allow accurate measurement of corneal thickness or iris volume, it does not natively allow measurement of PCAL. The software used in this project was newly developed as part of the study and is something which we now utilize in our own clinical practice when planning endothelial keratoplasty.

The use of anterior segment OCT imaging to facilitate the pre, peri, and post-operative assessments of patients undergoing anterior segment surgery is already well documented. ${ }^{11-13}$ Tan et al. ${ }^{14}$ showed there to be good correlation between PCAL measured using AS-OCT (time-domain) and intra-operative graft trephine diameter. AS-OCT based post operative determination of DSAEK graft diameter was also shown to correlate accurately with PCAL and actual trephine size. Pre-operative estimation of PCAL should allow surgeons to determine optimal graft diameter based on internal metrics of each patients eye. Our data suggests that PCAL correlates extremely well with WTW measurements, with PCAL being on average $1.82 \mathrm{~mm}$ longer. From our results, one can infer that, as a rule of thumb, PCAL is 1.16 times greater than the WTW.

Most surgeons performing Descemet stripping automated endothelial keratoplasty (DSAEK) measure donor graft size based on the WTW measurement, and our findings suggest that this method will under-estimate the posterior corneal surface available for endothelial cell transplantation. In our images below Figure 1 we illustrate this by showing how much posterior corneal arc length $8 \mathrm{~mm}$ and $8.5 \mathrm{~mm}$ grafts would cover when transplanted into eyes with differing PCAL measurements. It is clear that even in an eye with a relatively short PCAL (in this case our shortest recorded was $11.53 \mathrm{~mm}$ ) there is significant room for a larger graft.

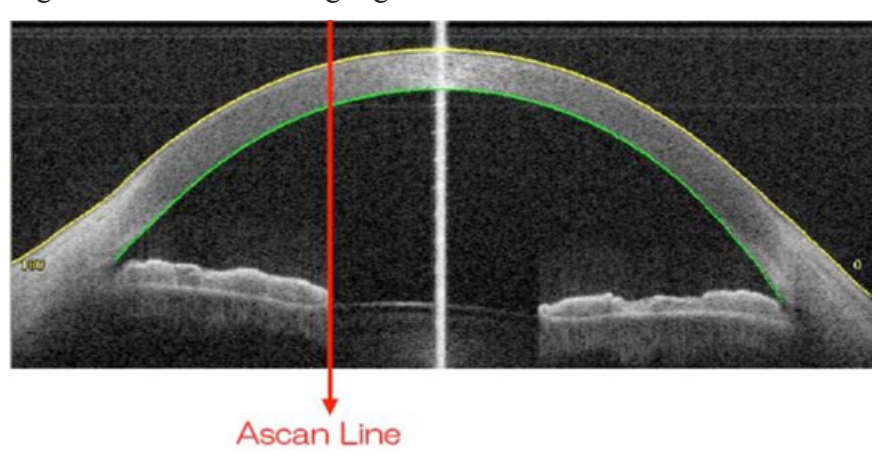

Figure I Sample OCT image showing software plotting of anterior (yellow) and posterior (green) corneal curvatures.

Larger DSAEK grafts have recently been shown to result in better graft survival, possibly due to a greater number of transplanted endothelial cells. ${ }^{1}$ In contrast Anshu et al. ${ }^{15}$ published data which suggested that a larger graft diameter would provide a larger reservoir of healthier endothelial cells but did not result in improved endothelial cell survival. Terry et al. ${ }^{16}$ retrospectively compared $8.0 \mathrm{~mm}$ and $8.5 \mathrm{~mm}$ DSAEK grafts in patients with Fuch's endothelial dystrophy over a 2 year period. They too suggested that a larger endothelial graft did not offer a clinical advantage with regard to post-operative endothelial cell counts. However, both of these papers use endothelial cell density (cells $/ \mathrm{mm} 2$ ) as an outcome measure. It would stand to reason that the diameter of the graft has no affect on the endothelial density, but it would seem reasonable that transplanting a larger graft, with a therefore greater total number of endothelial cells, would elongate long-term graft survival. The correlation of long term graft survival based and initial diameter is yet to be fully established.

The ability to accurately measure PCAL may be of particular importance in choosing a graft size in Descemet Membrane Endothelial Keratoplasty (DMEK) surgery where endothelial cells and Descemet membrane are transplanted without stroma. In 2012, Schlomberg et al. ${ }^{17}$ stated that complete removal of descemet membrane in the area of the graft allows better adhesion suggesting that a greater overlap of the graft over the area of descemetorrhexis was a risk factor leading to graft detachment. DMEK surgeons now intentionally under-size the donor graft scrolls to avoid overlap with edges of the descmetorrhexis with evidence that subsequent cell migration bridges the denuded gap over time. ${ }^{18}$ In this context, knowledge of PCAL may help surgeons to size the donor graft diameter more accurately based on PCAL rather than simply under-sizing the graft compared to the cord length diameter of a surface corneal mark.

\section{Conclusion}

Our data suggests that PCAL correlates well with WTW measurement in a British Caucasian population. The ratio of WTW to PCAL was 1.16 and this multiplication factor may be used at least in this patient cohort to estimate PCAL from per-operative WTW measurement. To be accurate, further analysis of larger patient numbers and demographics comparing WTW and PCAL measurements would be necessary. We have developed efficient and simple software for accurately measuring posterior corneal arc length. This is the first data documenting PCAL measurements in a White Caucasian population. PCAL may be a valuable utility for optimal sizing of endothelial grafts but further analysis and larger patient numbers are required.

\section{Funding}

None.

\section{Acknowledgments}

We thank Kathrin Hoffman and the team at Tomey Inc for their assistance in developing the image analysis software.

\section{Conflicts of interest}

The authors have no conflicts of interest to declare.

\section{References}

1. Vito Romano, Adrian Tey, Natalie M E Hill, et al. Influence of graft size on graft survival following Descemet stripping automated endothelial keratoplasty. Br J Ophthalmol. 2015.

2. Huang D, Swanson EA, Lin CP, et al. Optical Coherence Tomography. Science. 1991;254(5035):1178-1181.

3. Bianciotto C, Shields CL, Guzman JM, et al. Assessment of Anterior Segment Tumours with ultrasound biomicroscopy versus anterior segment optical coherence tomography in 200 cases. Ophthalmology. 2011;118(7):1297-3024.

4. Matonti F, Chazalon E, Trichet E, et al. Dynamic gonioscopy using optical coherence tomography. Ophthalmic Surg Lasers Imaging. 2012;43(6 Suppl): S90-S96. 
5. Scorcia V, Busin M, Lucisano A, et al. Anterior segment optical coherence tomography-guided big-bubble technique. Ophthalmology. 2013;120(3):471-476.

6. Console JW, Sakata LM, Aung T, et al. Quantitative analysis of anterior segment optical coherence tomography images: the Zhongshan Angle Assessment Program. Br J Ophthalmol. 2008;92(12):1612-1616.

7. Tan DK, Chong W, Tay WT, et al. Anterior chamber dimensions and posterior corneal arc length in Malay eyes: an anterior segment optical coherence tomography study. Invest Ophthalmol Vis Sci. 2012;53(8):4860 4867 .

8. Yuen LH, He M, Aung T, et al. Biometry of the cornea and anterior chamber in Chinese eyes: an anterior segment optical coherence tomography study. Invest Ophthalmol Vis Sci. 2010;51(7):3433-3440.

9. Ang M, Chong W, Tay WT, et al. Anterior segment optical coherence tomography study of the cornea and anterior segment in adult ethnic South Asian Indian eyes. Invest Ophthalmol Vis Sci. 2012;53(1):120-125.

10. Choma M, Sarunic M, Yang C, et al. Sensitivity advantage of swept source and Fourier domain optical coherence tomography. Optics Express. 2003;11(18):2183-2189.

11. Nguyen P, Chopra V. Applications of optical coherence tomography in cataract surgery. Curr Opin Ophthalmol. 2013;24(1):47-52.
12. Wylegala E, Dobrowolski D, Nowińska A, et al. Anterior segment optical coherence tomography in eye injuries. Graefes Arch Clin Exp Ophthalmol. 2009;247(4):451-455

13. De Benito-Llopis L, Mehta JS, Angunawela RI, et al. Anterior Segment Optical Coherence Tomography: A Novel Assessment Tool During Deep Anterior Lamellar Keratoplasty. Am J Ophthalmol. 2013;157(2):334-341.

14. Gavin S Tan, Mingguang He, Donald T Tan, Jodhbir S Mehta. Correlation of anterior segment optical coherence tomography measurements with graft trephine diameter following descemet stripping automated endothelial keratoplasty. BMC Med Imaging. 2012;12:19.

15. Anshu A, Price MO, Price FW Jr. Descemet stripping automated endothelial keratoplasty for Fuchs endothelial dystrophy-influence of graft diameter on endothelial cell loss. Cornea. 2013;32(1):5-8.

16. Terry MA, Li J, Goshe J, Davis-Boozer D. Endothelial keratoplasty: the relationship between donor tissue size and donor endothelial survival. Ophthalmology. 2011;118(10):1944-1949.

17. Schlomberg J, Tourtas T, Riss S, et al. Extent of removal of descemet membrane determines graft adhesion in DMEK. 2012.

18. Jacobi C, Zhivov A, Korbmacher J, et al. Evidence of endothelial cell migration after descemet membrane endothelial keratoplasty. Am J Ophthalmol. 2011;152(4):537-542. 